\title{
Una propuesta de enseñanza para los estudiantes de grado once del I. E. D. Paulo Freire sobre la noción de límite ${ }^{1}$
}

\author{
Um ensinamento propostas alunos do décimo primeiro \\ de I. E. D. Paulo Freire sobre a noção de limite
}

A proposed teaching eleventh grade students of

I. E. D. Paulo Freire on the notion of limit

Recibido: mayo de 2013

Aceptado: agosto de 2013
Anderxon Fabián Olaya Duran ${ }^{2}$

Torres Mondragón Karen Yissed ${ }^{3}$

Parra López Ginneth Viviana ${ }^{4}$

Milton Jefferson Villamil Camelo ${ }^{5}$

\section{Resumen}

El siguiente documento presenta una secuencia de actividades para trabajar la noción del concepto de limite involucrado en el pensamiento variacional en grado once, donde se toma como punto de partida el trabajo con sucesiones, permitiendo desarrollar a través del uso de diferentes tipos de sucesiones y la noción de convergencia; dicho concepto, tomado desde la definición de (Steward, Redlin, \& Watson, 2001). Basado en la metodología propuesta por el grupo (DECA, 1992), la cual, no solo muestra el enseñar matemáticas, como entregar algoritmos al estudiante, sino que por el contrario, un aprendizaje desde la construcción del objeto matemático, resaltando la participación activa y critica del estudiante.

Palabras clave: Enseñanza; metodología de enseñanza; secuencia de actividades; DECA; sucesiones; convergencia; matemáticas escolares; cálculo; límites; noción de límite.

\begin{abstract}
\end{abstract}
The following paper presents a sequence of activities to work the notion of the concept of limit involved in thinking variational juniors, which takes as its starting point the work sequences, allowing to develop through the use of different types of inheritance and the notion of convergence that concept, taken from the definition of (Stewart, Redlin, \& Watson, 2001). Based on the methodology proposed by the group (DECA, 1992), which not only shows the mathematics teaching as algorithms provide the student, but on the

1 Artículo de Investigación.

2 Universidad Distrital Francisco José De Caldas. Bogotá, Colombia. Contacto: afo.udistrital@gmail.com

3 Universidad Distrital Francisco José De Caldas. Bogotá, Colombia. Contacto: karentorres24@hotmail.com

4 Universidad Distrital Francisco José De Caldas. Bogotá, Colombia. Contacto: ginneth1226@hotmail.com

5 Universidad Distrital Francisco José De Caldas. Bogotá, Colombia. Contacto: miltonvillamil@hotmail.es 
contrary, learning from the construction of the mathematical object, highlighting the active and criticizes the student.

Keywords: Education, teaching methodology, sequence of activities, DECA; succession; convergence school math, calculus, limits; notion of limit.

\section{Resumo}

$\mathrm{O}$ artigo a seguir apresenta uma seqüência de atividades para trabalhar a noção do conceito de limite envolvido em pensar juniores variacionais, que toma como ponto de partida as seqüências de trabalho, permitindo a desenvolver através do uso de diferentes tipos de herança e noção de que o conceito de convergência, feita a partir da definição de (Stewart, Redlin, e Watson, 2001). Com base na metodologia proposta pelo grupo (DECA, 1992), que mostra não só o ensino da matemática como algoritmos proporcionar ao aluno, mas, pelo contrário, a aprendizagem a partir da construção do objeto matemático, com destaque para o ativo e critica o estudante.

Palavras-chave: educação, metodologia de ensino, seqüência de atividades, DECA; sucessão; matemática escolar convergência, cálculo, limites, noção de limite.

\section{Contextualización}

Esta experiencia se desarrolla durante la práctica intermedia III en el colegio I. E. D. Paulo Freire en el ciclo de educación media vocacional, enfocado a la construcción de una primera noción de Límite. Para comenzar el abordaje al objeto matemático escolar, se hace uso de los Estándares de Competencias para matemáticas (M E N, 2006), centrado en el pensamiento variacional y sistemas analíticos. En este trabajo se presenta una propuesta de aula, que se obtuvo a partir de la planeación, diseño y gestión de la secuencia de actividades que se planteó para la comprensión y aproximación a la noción de Limite, en donde a partir de las dificultades y logros evidenciados, se planeaban y elaboraban las próximas actividades, que permitieran el aprendizaje hacia el objeto matemático que se pretendía trabajar.

\section{Referentes teórico - prácticos básicos}

Se parte de la visón amplia que la variación es la matemática en el movimiento, es decir se deja de lado la matemática estática y se le da paso a una matemática moderna, donde se puede ver expresiones algebraicas que modelan dichos movimientos, donde los diferentes pensamientos se ponen en juego, ya sea al geométrico para medir, el numérico para calcular, entre otros que son de vital importancia y que de esta forma construyen un pensamiento, que da paso a los grandes descubrimientos del mundo, como lo son el cálculo infinitesimal e integral.

Desde los Lineamientos Curriculares se centra una mirada al desarrollo del pensamiento variacional y sistemas algebraicos y analíticos, en especial a cada una de las herramientas que ofrece para el desarrollo; presupone superar la enseñanza de contenidos matemáticos fragmentados y compartimentalizados, para ubicarse en el dominio de un campo conceptual, que involucra conceptos y procedimientos interestructurados $y$ vinculados que permitan analizar, organizar y modelar matemáticamente situaciones y problemas. (MEN, 1998).

Para ello, se ha retomado el trabajo de paradojas en matemáticas, como son las de Zenón, donde se utilizó la de Aquiles y la tortuga. Estas nos abren el camino directamente a las sucesiones, ya que es una forma numérica de presentar las diferentes situaciones planteadas por los Estudiantes Para Profesor (EPP). Hablando en general, una sucesión o progresión es una lista de números escritos en un orden específico, y una serie es lo que se obtiene sumando los números de una sucesión. (León, 2001). 
La sucesión la encontramos definida como:

"Función f cuyo dominio es el conjunto de los números naturales. Los valores $\mathrm{f}(1), \mathrm{f}(2)$, $\mathrm{f}(3), \ldots$, se llaman términos de la sucesión." (Steward, Redlin, \& Watson, 2001).

Observando los diferentes tipos de sucesiones que se van a trabajar, las cuales son: Finita o infinita, acotadas inferiormente, acotadas superiormente, acotadas, divergentes y convergente.

Teniendo en cuenta los anteriores tipos de sucesiones se hace referencia a las sucesiones divergentes y convergentes, ya que el objetivo principal es llegar una primera noción de límite.

El límite de una función $\mathrm{f}(\mathrm{x})$, cuando $\mathrm{x}$ tiende a c es L si y sólo si para todo existe un tal que para todo número real $\mathrm{x}$ en el dominio de la función . (Steward, Redlin, \& Watson, 2001).

Ahora, teniendo en cuenta que tenemos una noción de límite de una sucesión, se hace necesario el paso de sucesión a función; es decir que se pueda aplicar el concepto de límite, en funciones. Para realizar ese paso se utilizan las diferentes representaciones haciendo que las gráficas del objeto matemático sean las idóneas en el momento de comprender el lenguaje matemático, haciendo que el estudio de las mismas sea más práctico y entendible. (Azcarate, 1996). De esta forma también se hace necesario la diferencia de notación entre las sucesiones y funciones. (Steward, Redlin, \& Watson, 2001).

\section{Descripción}

Estas actividades están propuestas bajo la metodología el grupo (DECA, 1992).En cuanto a la fase de reestructuración, se diseñaron dos actividades, en la primera se hace relevancia a la ubicación de los diferentes conjuntos numéricos en la recta numérica, en la segunda se utilizan técnicas de aproximación en procesos infinitos numéricos, por medio de paradojas de Zenón.
Para la fase de profundización, se realizaron dos sesiones de clase. En la primera se pretendía generalizar las sucesiones y fomentar el razonamiento inductivo. En la segunda actividad se trabajan sucesiones convergentes y divergentes, con el trabajo de la primera, se buscaba llegar a una primera noción de límite; luego se realiza una actividad donde la temática es el límite de una sucesión y el propósito es que los estudiantes formalicen la noción de límite que se pretendía enseñar. Otra de las actividades realizadas, es "funciones parecido a sucesiones", donde la temática principal es diferenciar las sucesiones de las funciones. Por último, en la actividad de evaluación, el propósito era hacer un recuento de lo presentado en la secuencia: generalización de sucesiones, sucesiones convergentes y divergentes, y la diferencia de las funciones y sucesiones teniendo en cuenta la relación que existe entre ellas y que coinciden con la noción de límite. En donde a través de una guía de trabajo, el estudiante demuestra lo aprendido de dichas temáticas y resuelve situaciones problema por medio de dichos aprendizajes.

\section{Logros y dificultades alcanzadas}

Con la aplicación de la secuencia de actividades, y teniendo en cuenta que con ellas se hizo un abarcamiento de la representación de los números reales en la recta numérica, pasando por el trabajo de las paradojas de Zenón, las sucesiones, y las funciones, con el fin de abordar el objeto matemático planteado de la noción de limite; se logró evidenciar en primera instancia, que la propuesta de trabajo, generó una gran acogida por el grupo de estudiantes del grado once, que como mostró la prueba diagnóstico era un tema un poco complejo para su entendimiento.

En cuanto a lo conceptual se puede afirmar que los estudiantes construyeron una primera noción de límite, esto se dio gracias a las diferentes sucesiones que se trabajaron, donde la ubicación en la recta numérica, fue la base de este proceso de enseñanza aprendizaje. Por otro lado, se entendió de manera gráfica la diferencia entre sucesión y función, avanzando paralelamente en el apropiamiento del lenguaje matemático y de la noción de límite en cada una de estas. Frente a las dificultades identificadas, 
se puede hacer referencia que en el momento de llevar a cabo la aplicación de esta propuesta, la institución educativa no cuenta con la tecnología (calculadoras graficadoras - cabri), que permita una mejor construcción de la noción de límite. Además se identificó en la prueba diagnóstico que los estudiantes cuentan con pocas bases para enfrentar este proceso cognitivo; siendo la comprensión de lectura uno de los más visibles.

Es importante que los estudiantes estén familiarizados con el concepto de las distintas interpretaciones de la letra en el álgebra, para tener una mayor comprensión en la introducción del concepto de función lineal. Además es pertinente que todo objeto matemático que se enseñe en la escuela deba ser contextualizado a la realidad socio-política-cultural de los estudiantes.

\section{Reflexión final}

Con el desarrollo de la presente experiencia de aula que buscaba proponer una mirada hacia el cálculo infinitesimal, se puede inferir que se logró presentar una serie de actividades que permitieron incluir el pensamiento variacional en grado once partiendo del trabajo con sucesiones, donde se le permitió al estudiante construir y elaborar una noción de convergencia y una primera noción de límite; gracias al uso de una metodología de enseñanza basada en el grupo (DECA, 1992), se mostró que enseñar matemáticas, no significa simplemente entregar algoritmos al estudiante para que los memorice, sino que por el contrario, se debe priorizar las acciones del estudiante con el fin de generar un aprendizaje desde la construcción propia que realiza frente al objeto matemático.
Estamos convencidos que la educación, necesita docentes, con una postura diferente, que motive, estimule y genere estrategias para el proceso de enseñanza - aprendizaje; docentes comprometidos con una educación de calidad, que este en contante investigación, sobre las "novedades de la educación", un docente capaz de gestionar propuestas interesantes y eficaces sobre dicho proceso.

\section{Referencias}

Azcarate, C. (1996). Funciones y gráficas. España: sintesis.

DECA. (1992). Orientaciones para el diseño y elaboración de actividades de aprendizaje y de evaluación. Aula (6), 33 - 39.

León, C. P. (24 de 06 de 2001). http://serbal. pntic.mec.es/ cmunoz11/paradojas.pdf. Recuperado el 03 de 04 de 2011, de http:// serbal.pntic.mec.es/ cmunoz11/paradojas. pdf: http://serbal.pntic.mec.es/ cmunoz11/ paradojas.pdf

M E N. (2006). Pensamiento Variacional y sistemas algebráicos y analíticos. Bogotá: Magisterio.

MEN. (1998). Lineamientos curriculares para el área de matemáticas. Bogotá: Magisterio.

Steward, J., Redlin, L., \& Watson, S. (2001). Precálculo. Colombia: Thomson Editores S.A. 